\title{
Aparición y desarrollo de la atención conjunta en la infancia
}

\author{
Alfonso Escudero-Sanz ${ }^{1 *}$, José A. Carranza-Carnicero ${ }^{1}$ y Elisa Huéscar-Hernández ${ }^{2}$ \\ ${ }^{1}$ Universidad de Murcia. Departamento de Psicología Evolutiva y de la Educación \\ ${ }^{2}$ Universidad Miguel Hernández de Elche, Departamento de Psicología de la Salud
}

\begin{abstract}
Resumen: La Atención Conjunta constituye la primera condición sobre la que se construye la comunicación. De ahí el enorme interés que despierta su estudio, dada su influencia sobre el desarrollo cognitivo, social, emocional, y lingüístico humano. Este artículo presenta una revisión de la investigación sobre la aparición y el desarrollo de la Atención Conjunta en la infancia, poniendo de relieve los principales elementos de debate sobre dicha temática. Comenzamos examinando el concepto de intencionalidad en la definición de la Atención Conjunta, para describir a continuación la secuencia de desarrollo de dicha capacidad. Finalizamos el trabajo ofreciendo algunos datos que relacionan la Atención Conjunta con el desarrollo del lenguaje, y sugiriendo direcciones en las que se puede orientar la investigación en este campo.
\end{abstract}

Palabras clave: Atención conjunta; interacción madre-hijo; lenguaje; infancia.

\section{Introducción}

Los niños nacen en el seno de un mundo social, y crecen como seres sociales, inmersos desde el principio en una red de relaciones con los demás (Schietecatte, Roeyers, y Warreyn, 2011). Por ello, la interacción es el mecanismo básico del desarrollo humano. A su vez, la comunicación es la base de toda interacción social.

Comunicación proviene de la palabra latina communis, que significa común, por lo que comunicarse requiere ponerse de acuerdo sobre aquello de lo que se trate. Una manera de asegurar este acuerdo es compartir el mismo centro de atención. Así pues, la interacción exige algún mecanismo que garantice que el foco de interés de los interlocutores es el mismo (Ricciardelli, Betta, Pruner y Turatto, 2009).

Pero no basta con atender al mismo objeto. Una secuencia de interacción social se caracteriza por una sucesión de acciones y reacciones de cada uno de los interlocutores. Dicha secuencia exige una coordinación entre los interlocutores que les permita adaptar su conducta a la respuesta del otro. Pero cuando la interacción tiene lugar con un niño pequeño, es el adulto el que inicialmente asume la mayor parte de responsabilidad en el mantenimiento de dicha coordinación (Sun y Rao, 2012), si bien el niño no es completamente pasivo: desde el momento de nacer, dispone de un bagaje biológico en forma de capacidades perceptivo-motoras y afectivo-emocionales que le permitirán establecer y mantener esos intercambios sociales (Ricciardelli, Betta, Pruner y Turatto, 2009). Se trata de lo que Trevarthen (1990) denominó intersubjetividad primaria, que tiene lugar entre los dos y los cuatro meses. Para Trevarthen la intersubjetividad es el proceso de compartir un significado. En un primer momen-

\section{* Dirección para correspondencia [Correspondence address]}

Alfonso Escudero Sanz. Departamento de Psicología Evolutiva y de la Educación, Facultad de Psicología, Universidad de Murcia, Campus de Espinardo, 30100, Murcia, España. E-mail: aescuder@um.es
Title: Emergence and development of Joint Attention in infancy. Abstract: Joint Attention is the first step to build communication. Hence, its study is of great interest due to its influence on cognitive, social, emotional, and language development. This article presents a review of the research on the emergence and development of Joint Attention in infancy, highlighting the major points of discussion on this topic. We begin by examining the concept of intentionality in the definition of Joint Attention, followed by a description of the sequence of development of this capacity. We finish relating joint attention to language development and suggesting directions that can guide ulterior research in the field.

Keywords: Joint attention; mother-child interaction; language; children.

to, el significado que se comparte tiene un carácter emocional y automático: el bebé responde a las expresiones afectivas de los adultos. Cuando lo hace, en cierta medida las reconoce y, además, las diferencia de las respuestas que da a otros estímulos no sociales. La aparición de los protodeclarativos en el último tercio del primer año indica el comienzo de la verdadera intención comunicativa y de la capacidad de relacionarse con las personas como entidades diferentes del "yo". En opinión de Trevarthen, el niño manifiesta partir de ese momento una intersubjetividad secundaria, es decir, una motivación deliberada por compartir los intereses y las experiencias con otras personas.

Así pues, si durante el primer semestre el bagaje innato del bebé asegura una comunicación básica de carácter emocional, centrada en sí mismo y dirigida por el adulto, una vez que los objetos se convierten en los catalizadores de la interacción, se desencadenan una serie de cambios cognitivos, sociales, y lingüísticos que ejercerán profundas consecuencias para el desarrollo posterior del niño (Olson y Masur, 2011). Como ha sugerido Lois Bloom (1993), a medida que nuestro mundo mental se separa de nuestro mundo perceptivo, necesitamos mecanismos que nos permitan establecer un puente entre ambos. En el mismo sentido, Clark (2009) ha señalado que los participantes en una conversación necesitan observar cierto número de condiciones para que la comunicación sea eficaz, lo que implica que deben:

- compartir un centro de atención

- tener en cuenta el conocimiento de su interlocutor y adaptar su lenguaje a ese conocimiento

- elegir actos de habla apropiados para los significados que intentan transmitir

- escuchar lo que dicen los otros para poder hacer contribuciones apropiadas cuando les llegue el turno.

Así pues, la atención conjunta constituye la primera condición sobre la que se construye la comunicación. Como ha 
señalado Liszkowski (2011) para poder adquirir cualquier código arbitrario, como es el lingüístico, es imprescindible disponer de capacidades comunicativas que permitan comprender lo que se está codificando. De ahí el enorme interés que despierta su estudio, así como su influencia sobre la adquisición del lenguaje, y el desarrollo cognitivo, social, emocional, y lingüístico humano (Blacher y Lauderlade, 2010; Bruner, 1975, 1977; Meins et al., 2011; Tomasello, 1988, 1995).

\section{Atención conjunta e intencionalidad}

Si quiero comunicarme contigo, debo asegurarme de delimitar claramente el referente que deseo compartir. ¿Significa eso que actúo de manera intencional? La respuesta que se dé a esa pregunta modifica de manera sustancial la definición de la Atención Conjunta, y de hecho, podríamos establecer una línea divisoria entre los dos tipos de definiciones (según la naturaleza no intencional versus intencional) que hasta hoy ofrece la literatura sobre esta cuestión, y que encajarían, respectivamente, con el desarrollo evolutivo de la intersubjetividad infantil propuesto por Trevarthen.

Así pues, en una primera etapa (intersubjetividad primaria) el niño adopta un papel pasivo en la interacción, de manera que es el adulto el que promueve las situaciones de atención conjunta. Se apoya para ello en indicadores verbales como "¡mira!", o en la dirección de la mirada (Estigarribia y Clark, 2007). A su vez, el niño responde a la iniciativa del adulto merced a una serie de mecanismos innatos. Inicialmente, dichas respuestas son simples y sólo se ponen en marcha en el seno de rutinas y situaciones de interacción muy estructuradas. Progresivamente, el niño va adquiriendo una capacidad cada vez más refinada para responder a pistas más sutiles, aunque todavía dependientes del adulto. Mientras que en las conversaciones entre adultos los interlocutores comprueban sistemáticamente a qué está atendiendo cada uno, y se coordinan entre sí para lograr la atención conjunta, la interacción entre un adulto y un niño pequeño es muy asimétrica, pues es el primero el que carga con la dirección y el mantenimiento de la misma. (Leong y Bodrova, 2012; Tomasello 1995). En esta fase de intersubjetividad primaria, los adultos se basan fundamentalmente en claves perceptivas para saber a qué está atendiendo el niño: siguen la dirección de su mira$\mathrm{da}$, e interpretan cualquier indicador disponible, como puede ser la orientación de su cuerpo (Clark, 1997; Dueker, Portko, y Zelinsky, 2011; Yoshida y Burling, 2011).

Dado que la combinación del bagaje innato del niño, junto con el interés del adulto por comunicarse, facilitan la convergencia de su atención, algunos autores consideran suficiente que se produzca la coincidencia entre ambas miradas, sin tomar en consideración si dicha conjunción es intencionada o no. Por ejemplo, Butterworth (1991) define la Atención Conjunta como la capacidad de seguir la dirección de la mirada del otro, o "mirar donde alguien más está mirando" (p. 223). Idéntica es la definición de Emery, Lorinz, Perret, Oram, y Baker, (1997), como la conducta de seguir la direc- ción de la mirada de otra persona hacia un objeto determinado al que ésta última está atendiendo. En definitiva, desde esta perspectiva basta con que el niño observe el giro de cabeza del adulto y se vuelva para mirar en la misma dirección, para que consideren que nos encontramos ante un episodio de Atención Conjunta (Ricciardelli, Betta, Pruner y Turatto, 2009). En la actualidad está tomando fuerza la hipótesis de la "personificación" (embodiment), que destaca el hecho de que la cognición, lejos de desarrollarse de manera autónoma y meramente simbólica, necesita a un organismo activo que interactúe con su entorno para la solución de problemas (Dueker, Portko, y Zelinsky, 2011). Más allá de la capacidad de procesamiento, es muy importante el papel activo del sujeto en el proceso de interacción.

Para otros autores, sin embargo, este tipo de episodios atencionales no pasan de ser un tipo de intercambio fortuito, que han denominado atención "pasiva" o "apoyada", y donde la responsabilidad de la interacción recaería totalmente sobre el adulto (Bakeman y Adamson, 1984). Por lo demás, la transición desde estas respuestas no intencionales hacia conductas más coordinadas y deliberadas por parte del niño, exige de éste una mayor madurez que la implícita en las definiciones anteriores.Términos como "coordinación" (Tomasello, 1995), "verificación” (Mundy, Hogan y Doehring, 1996), o "reconocimiento" (Baldwin, 1995), pretenden etiquetar esta nueva capacidad del niño para adaptar su atención a la de los demás. Así pues, ahora el niño ya sabe que el otro está mirando algo que coincide con lo que él está contemplando (Mundy y Gomes, 1998; Mundy y Sigman, 2006). En este sentido, Campbell (2002) afirma que la Atención Conjunta debe estar recíprocamente supervisada: esto es, ambos interlocutores tienen que estar pendientes de lo que está atendiendo la otra persona y además los dos participan activamente en esta labor. Dichas propuestas se han visto confirmadas recientemente por investigaciones (Chanon y Hopfinger, 2011) que han comparado los potenciales evocados producidos por la atención refleja a un estímulo llamativo, la atención voluntaria, y la atención involuntaria que sigue la mirada de otra persona. Sus resultados demuestran que, pese a su similitud conductual, el seguimiento visual de la mirada de una persona produce diferentes efectos neurológicos que la atención refleja a un estímulo. Por el contrario, los potenciales evocados producidos por dicho seguimiento visual de la mirada, fueron muy similares a los que se produjeron durante la atención voluntaria.

En este punto, según Adamson y MacArthur (1995), la introducción de los objetos en la interacción supone un punto de inflexión en esta secuencia de desarrollo, dado que ahora los niños desean dirigir la atención del adulto hacia éstos. Avanzarán así hacia una conducta cada vez más manifiestamente intencional y activa. Para estos autores, los componentes que forman parte de estos episodios interactivos serían: el bebé, el cuidador, los objetos situados en la proximidad inmediata, y los elementos simbólicos presentes en los códigos convencionales del habla (Liebal, Carpenter, \& Tomasello, 2010). Por ejemplo, MacCune (2008) sugiere que dichos 
elementos determinan la comunicación interpersonal entre el niño y el adulto. En esencia, la comunicación procede de la necesidad de mantener el contacto con el adulto, y se establece inicialmente a partir de elementos no simbólicos como la atención conjunta y la señalización.

Así pues, esta deliberada motivación para compartir con los demás los objetos de su interés, señala el comienzo de la intersubjetividad secundaria, y acentúa el papel de la intencionalidad. Por lo tanto, desde esta perspectiva cognitiva, para que se establezca la Atención Conjunta ya no basta con que los interlocutores miren simultáneamente a la misma entidad, sino que además deben ser conscientes de que comparten un centro de interés (Liszkowski, 2011). Por ejemplo, Olson y Masur (2011) han demostrado que la presencia de objetos en la interacción provoca, por parte de los niños prelingüísticos, gestos diferentes para expresar sus intenciones hacia los mismos. Suelen señalar con una intención predeclarativa, y extender los brazos hacia el objeto con intención preimperativa. Pues bien, las madres actuaban en consecuencia, y expresaban más nombres en respuesta a las señalizaciones, y más etiquetas de acción como respuesta a la extensión de brazos hacia los objetos. Así, una vez que los objetos pasan a formar parte de la interacción entre el bebé y el adulto, se pone en marcha una eficaz red de significados compartidos que pueden servir, cuando menos, para la adquisición del vocabulario.

De esta manera, alrededor de su primer cumpleaños, el niño ya es capaz de coordinar con su interlocutor, de manera activa e intencional, su atención hacia un mismo objeto, y además ambos saben que comparten ese mismo interés (Hobson, 1989). Tomasello (Gerlind y Tomasello, 2012; Grassmann y Tomasello, 2010; Tomasello, 1995; Tomasello, Carpenter, Call, Behne, y Henrike, 2005; Tomasello y Carpenter, 2007) se ha convertido en uno de los principales referentes de la concepción cognitiva de la Atención Conjunta. Su propuesta básica es que para que los participantes en una interacción puedan compartir una misma meta, deben concentrarse en el mismo objeto y además estar atentos a los comportamientos del interlocutor para poder acomodarse a él (1995, pp. 105-107):

ambos participantes están controlando la atención del otro hacia la entidad externa... y la coordinación que tiene lugar en las interacciones de atención conjunta se alcanzan gracias a la comprensión de que el otro participante está concentrando su atención sobre la misma entidad que uno mismo

Como vemos, desde esta perspectiva la Atención Conjunta va más allá de una simple coincidencia de la mirada de los dos participantes en la interacción, y requiere la presencia de una serie de conductas activas por parte del niño. Esta transición no se produce, según Tomasello (1995), hasta aproximadamente los 12 meses de edad, momento en que los niños se consideran ya a sí mismos y a los demás como seres intencionales, lo que coincide evolutivamente con la aparición de las habilidades simbólicas (Baron-Cohen, 1994; Hobson, 1993).

A partir de este componente de reciprocidad implícito en la Atención Conjunta, Kaplan y Hafner, (2004) proponen una serie de requisitos para que ésta tenga lugar:

\section{Detección de la atención}

Un agente debe ser capaz de seguir la orientación de la atención de otros agentes, por ejemplo, la dirección de la mirada del otro. Coincide con la definición de Atención Conjunta de los estudios pioneros (Butterworth, 1991; Emery, Lorinz, Perret, Oram, y Baker, 1997).

\section{Coordinación social}

Los interlocutores deben implicarse en una interacción coordinada. Esto supone ya el manejo de estrategias más avanzadas de interacción social, tales como la adopción de turnos, el cambio de papeles, y juegos rituales como el cucú. Este requisito exige la capacidad de adoptar de un papel más activo, aunque todavía sea como mera respuesta a la iniciativa del adulto y no necesariamente con carácter intencional. Nos encontraríamos ante un tipo de interacción "pasiva" o "apoyada", como proponían Bakeman y Adamson (1984).

\section{Manipulación de la atención}

Los participantes deben ser capaces de manipular el comportamiento atencional de los otros copartícipes, manteniéndolo sobre el objeto de interés o cambiando el foco de atención hacia otro objeto o evento. Evidentemente, aquí juega un papel esencial el lenguaje y la señalización. La intencionalidad se hace ya patente, y se adopta una conducta activa. Tanto este requisito como el siguiente recogen la definición de Tomasello respecto a que la Atención Conjunta exige que los interlocutores estén atentos a los comportamientos del otro para acomodarse mutuamente a un mismo centro de interés.

\section{Posición intencional}

Los participantes deben considerarse a sí mismos como agentes intencionales. Deben entender que los otros tienen intenciones, posiblemente diferentes a las suyas. Al adoptar tal posición, interpretan y predicen el comportamiento de los demás asumiendo que está orientado hacia un objetivo (Dennett, 1987).

En definitiva, la Atención Conjunta evoluciona desde una coincidencia inconsciente de carácter emocional y administrada por el adulto, hacia una interacción plenamente intencionada por parte de todos los participantes, quienes intentan dirigir la conducta del interlocutor de manera activa y deliberada. Entre ambos extremos los niños avanzan desde una atención cada vez más sofisticada, pero pasiva y recepti- 
va, hacia un comportamiento más activo y recíproco dirigido hacia un objeto.

En el siguiente apartado profundizaremos sobre la secuencia evolutiva de la Atención Conjunta en sus aspectos más controvertidos, relacionados con su aparición y con las conductas que caracterizan la transición desde una interacción diádica a otra denominada triádica.

\section{Secuencia de desarrollo de la atención conjun- ta}

Como hemos visto, es posible encontrar antecedentes de la Atención Conjunta poco después del nacimiento, cuando el recién nacido y su cuidador comparten momentos de atención que normalmente no parten de un interés común, sino que surgen de la mera coincidencia de la atención que el adulto despliega hacia el niño y los episodios de alerta de éste.

Cuando el niño tiene entre 6-8 semanas de vida, comienza a desarrollarse la actividad interpersonal, entre otras cosas gracias a la reciprocidad afectiva con su cuidador durante sus cada vez más frecuentes interacciones cara a cara. Scaife y Bruner (1975) mostraron que los niños de dos meses de edad eran capaces de seguir la mirada de un adulto que se encontraba sentado frente a ellos, cuando éste giraba la cabeza hacia una señal luminosa situada a un lado de la habitación. Numerosas investigaciones han confirmado estos hallazgos (Butterworth y Hopkins, 1989; D'Entremont, Hains y Muir, 1997; Kalnins y Bruner, 1974; Lew y Butterworth, 1995, 1997; Morales, Mundy y Rojas, 1998; Von Hofsten, 1990). Los humanos estamos diseñados para seguir con eficacia la mirada de otra persona, y se ha demostrado que la atención a un objetivo determinado se produce de manera más rápida y precisa cuando ese objetivo está en la línea de mirada de otra persona (Driver, Davis, Ricciardelli, Kidd, Maxwell y Baron-Cohen 1999; Friesen y Kingstone, 1998). Aunque generalmente se ha considerado que esta atención a la dirección de la mirada tiene un importante componente reflejo, estudios muy recientes utilizando potenciales evocados demuestran que el seguimiento de la mirada produce potenciales evocados diferentes que la respuesta refleja, como ya habíamos señalado anteriormente (Chanon y Hopfinger, 2011). Así pues, la mirada aparece desde muy pronto como una manera muy eficaz de sintonizarse con el otro en una interacción.

Según Trevarthen (1990) a esta edad los niños ya manifiestan de manera innata ciertos propósitos comunicativos. Por ejemplo, Tremblay y Rovira (2007) encontraron que los niños de 3 y 6 meses ya son capaces de participar en una interacción social coordinada. Estos autores compararon el comportamiento de los bebés en dos experimentos: una condición de interacción persona-persona-persona (PPP) y otra condición compuesta por persona-persona-objeto (PPO). Únicamente en la condición PPP, en la que el niño observaba cómo el adulto que estaba enfrente de él mantenía una conversación con otro adulto, era capaz de participar y de mostrar su interés en la interacción a través de la mirada a uno y otro interlocutor. Según los autores, los niños preferían el escenario compuesto por tres personas (PPP frente a PPO) para participar (o mostrar interés) en la interacción, ya que en un primer momento las personas son elementos más familiares y atractivos para el niño que los objetos inanimados.

No obstante, a partir de los 5-6 meses, los bebés se interesan cada vez más por los objetos (Lamb, Morrison y Malkin, 1987; Messer y Vietze, 1984). Los episodios de actividad interpersonal se van haciendo más sostenidos e intencionados. Aunque los niños todavía no integran en su interacción actividades con los objetos, los cuidadores sí suelen fomentar de manera muy eficaz esta exploración, presentando los objetos de forma llamativa, o simplemente situándolos en el centro de atención. Este período, denominado de "inclusión del objeto" (Adamson y McArthur, 1995) se caracteriza por un fuerte apoyo del adulto para lograr que el niño se interese por ellos y los explore.

Durante este periodo, y hasta aproximadamente los 9 meses, no existe consenso respecto a cómo interpretar las incipientes habilidades de Atención Conjunta desplegadas por los niños. En esta línea, recientemente Grossmann y Jonhson (2010), han encontrado que cuando niños de cinco meses de edad interactúan con un adulto y un objeto, se activa su cortéx prefrontal izquierdo. Hace tiempo se conoce el estrecho vínculo que existe entre la orientación de la atención y los movimientos oculares (p.e. Hofman y Suramaniam, 1995; Awh et al., 2006 para una revisión). También se ha demostrado que los movimientos oculares comparten con la atención espacial los mismos mecanismos neuronales en la corteza parietal y prefrontal (Corbetta, 1998). En efecto, la corteza parietal y temporal superior está implicada en la imitación, la representación, y la percepción de la orientación de la cabeza y los ojos de los otros (Jellema, Baker, Wicker, y Perrett, 2000). Esta tendencia y los mecanismos neurológicos que la sustentan son comunes a muchos primates.

Así pues, aunque hacia los 6 meses de edad, los bebés sean capaces de orientarse en la misma localización espacial que los adultos, todavía no lo son de fijarse en las mismas características de los objetos que ellos miran, ni son conscientes del foco atencional compartido, por lo que no se podría hablar aún de atención conjunta en sentido estricto. Así pues, según Tomasello en este período sólo podríamos encontrar episodios que se basan en la mera coincidencia del foco atencional del niño y el adulto (Butterworth, 1991; Tomasello, 1998; Tomasello y Carpenter, 2007).

Entre los nueve y los doce meses, ya es posible observar los primeros indicios de que el niño es capaz de incluir un objeto en su interacción con el adulto (Cleveland y Striano, 2007). Según Adamson y McArthur (1995) los niños comienzan a tomar la iniciativa para promover la interacción. Lejos ya de la mera atención "asistida", impulsada y sostenida por el adulto, los niños empiezan a dirigir la atención y el comportamiento de su interlocutor, a manifestar sus prime- 
ras miradas alternas entre el adulto y el objeto, y a mostrar objetos al adulto sin que éste los haya solicitado.

También en este caso se han encontrado correlatos neuronales para tales conductas. El sistema atencional anterior controla la orientación voluntaria de la atención, e incluye las áreas de la corteza implicadas en el control de los ojos, la corteza prefrontal asociativa, la corteza orbital prefrontal, y el cíngulo anterior. Los estudios de neuroimagen y EEG han mostrado que los sistemas anterior y posterior se activan ante situaciones de atención conjunta (Mundy, 2003; Williams, Waiter, Perra, Perrett, y Whithen, 2005).

De esta manera, en la segunda mitad del primer año, el sistema atencional anterior va siendo capaz de integrar el control de la propia mirada con la dirección de la mirada de los demás, lo que permite a los niños (gracias a la maduración y la experiencia) comprender que ellos también tienen sus propias intenciones cuando dirigen su mirada hacia un objeto. Por otro lado, Dixon y Smith (2008), destacan que la maduración del sistema de atención anterior durante la segunda mitad del primer año, permite a los niños dirigir y mantener el foco de atención a su propia voluntad y por consiguiente, una distribución deliberada de la atención, jugaría un papel esencial en la predicción de la productividad lingüística posterior.

No obstante, sigue sin haber un consenso entre los investigadores en cuanto al grado en que tales conductas demuestran que el niño considera a los demás como agentes intencionales. Según Tomasello (1998), la mayoría de los comportamientos de atención conjunta que comienzan a manifestarse hacia los nueve meses, no se convertirán en claramente intencionales hasta, aproximadamente, los doce meses. Efectivamente, alrededor de primer año de vida aparecen el acto de señalar (Lempers, 1979; Carpenter et al., 1998; Corkum y Moore, 1998; Moore y Corkum, 1994; Morissette, Ricard, y Gouin-Decaire, 1995) y la referencia social, así como las rutinas para pedir objetos y referirse a los mismos (Adamson y McArthur, 1995).

La aparición de la conducta de señalar posee una importancia crucial durante este período, ya que refleja el tránsito desde un papel atencional pasivo y esencialmente receptivo, a otro más activo y directivo, cuyo objetivo es expresar sus intenciones al adulto. Señalar es a la misma vez una indicación que el niño sigue para delimitar el referente, pero también una manera de dirigir la atención de los otros. Si bien los niños son capaces de seguir la señal de otra persona a partir de los 9 o 10 meses de edad (Butterworth, 1991; Leung y Rheingold, 1981; Muñetón y Rodrigo, 2011; Murphy y Messer, 1977), sólo pueden hacerlo si los objetos están cercanos, y únicamente lograrán comprender las señales hacia objetos distantes cuando cumplan 14 o 15 meses de edad (Elgier y Mustaca, 2009; Morissette et al., 1995; Murphy y Messer, 1977). Además, se ha diferenciado entre señalar para pedir frente a señalar para mostrar (Bates, 1979; BaronCohen, 1995; Fenson, Dale, Reznick, Bates, Thal y Pethick, 1994; Tomasello, 1995). De esta manera, inicialmente, alrededor de los 12 meses de edad, los niños usan la conducta de señalar para pedir, y sólo posteriormente lo hacen para mostrar. Sin embargo, más recientemente Muñetón y Rodrigo (2011) han encontrado resultados diferentes: el señalamiento de mostrar tiene una mayor producción tanto en la madre como en el niño, y a nivel evolutivo emerge primero, seguido del de informar, pedir un objeto, pedir una acción y solicitar cooperación. Como señalan las propias autoras, probablemente esa discrepancia se deba al método de recopilación de datos: mientras que su estudio recurrió a un método naturalista en un contexto de interacción madrehijo, lo más habitual ha sido recurrir a métodos de laboratorio para provocar la señalización (Camaioni, 1993). Resulta interesante destacar que mientras que la señalización para mostrar genera un incremento del vocabulario en el niño, las peticiones de acción o de cooperación aumentan la atención conjunta entre madre e hijo, lo que destaca el papel fundamental de esta conducta en relación con diferentes aspectos de la interacción (Elgier y Mustaca, 2009; Tomasello, et al., 2007).

Sin embargo, a pesar del creciente número de estudios sobre este gesto, su naturaleza sigue siendo controvertida. Si bien al principio algunos autores supusieron que en realidad se trata de una conducta de alcance abreviada (Vygotsky, 1966; Werner y Kaplan, 1963), otros (Bates, 1979; Leung y Rheingold, 1981) han defendido que se trata de un gesto que aparece inicialmente dirigido a sí mismo, como para ayudarse a mantener su atención sobre un objeto, y que sólo posteriormente se utiliza para dirigir la atención de los demás. En definitiva, el gesto de señalar depende de una larga historia de aprendizaje entre el agente (niño) y el receptor (adulto). A medida que el niño tiene más oportunidades de interaccionar con el adulto va copiando la morfología y funciones de esta conducta. Por su parte, el adulto irá modelando y reforzando las iniciativas de señalización del niño (Leung y Rheingold, 1981).

En resumen, hemos visto que el niño evoluciona desde compartir fortuitamente la atención a seguirla activamente, para más tarde, en torno al primer cumpleaños, ser capaz de dirigir el interés de los demás hacia determinados objetos o eventos. A partir de este momento, coincidiendo con el comienzo del lenguaje, comienza a afianzarse la Atención Conjunta, aunque la verdadera consolidación de esta habilidad no se alcanzará hasta los 18 meses de edad. A partir de este momento, la atención conjunta adulto-niño-objeto deja de estar limitada por el espacio visual compartido cara a cara en el contexto de interacción. En efecto, mientras que hasta los doce meses los niños solo eran capaces de responder a situaciones con las personas y los objetos dentro de su campo visual, a partir de los dieciocho meses, también serán capaces de girarse para atender a objetos que se encuentren a su espalda, lo que constituye un nuevo avance en el desarrollo de este comportamiento. Hechos como este ponen de manifiesto el papel que juegan en el desarrollo de la atención conjunta otros procesos como los avances cognitivos y lingüísticos. 


\section{Relaciones de la atención conjunta con los inicios del lenguaje y la cognición}

Hemos visto que los niños nacen equipados para "comunicar" a los adultos sus sentimientos básicos, sintiéndose atraídos por los ojos y expresiones del adulto durante la interacción. Sin embargo, la presencia de un objeto en esa relación supone un salto cualitativo muy importante en dicho proceso. A partir del momento en que los objetos entran en escena, el adulto y el niño deben ser capaces de "referirse" a ellos.

Durante estos episodios de interacción, los adultos adaptan su lenguaje a los focos de interés de los niños. De esta forma, la atención conjunta ayuda a los pequeños a identificar el referente que el adulto está nombrando. Así por ejemplo, el gesto de señalar resulta determinante en el desarrollo simbólico y en el aprendizaje del lenguaje verbal (Goodwyn, Acredolo y Brown, 2000), ya que mediante este gesto el cuidador delimita el referente de una manera muy precisa. En este sentido, se ha demostrado una fuerte relación de los comportamientos de atención conjunta con la comunicación verbal posterior (Bates, Benigni, Bretherton, Camaioni y Volterra, 1979; Mundy, Kasari, Sigman y Ruskin, 1995; Olson, Bates y Bayles, 1984; Tomasello, 1995; Tomasello et al., 2007). Más específicamente, Kristen, Sodian, Thoermer, y Perst, (2011) han demostrado que la capacidad de atención conjunta a los 12 meses de edad predice el vocabulario.

La capacidad del niño para atender a lo mismo que está atendiendo su interlocutor no solo facilita la adquisición del vocabulario, sino también otros aspectos pragmáticos, sintácticos y fonológicos. Uno de los primeros autores que señaló la relación de la atención conjunta y el lenguaje temprano en los niños fue Bruner (1983), quien sostenía que era necesario estudiar de manera detallada todo el sistema de comunicación previo para poder entender la posterior adquisición del lenguaje (Bruner, 1975). Bates, Camaioni y Volterra (1975) y Bates (1979) habían señalado que la comunicación predeclarativa (dar, mostrar el objeto) del niño era un buen predictor del desarrollo léxico, y sugerían que podía ser el punto de partida de la comunicación referencial. También en esta línea, Butterworth (1991) había destacado la importante función comunicativa de la atención durante el período prelingüístico, debido a que proporciona información básica sobre los objetos con los que el niño interactúa.

No obstante, es importante puntualizar que el efecto facilitador de la atención conjunta puede verse muy influido por una variable fundamental en relación con el primer lenguaje, como es el estilo de los padres cuando interactúan con sus hijos. Se ha demostrado que si la madre sigue el foco de atención del niño cuando nombra los objetos que su hijo observa, existe una relación significativa y positiva con el desarrollo del léxico, pero si por el contrario, el cuidador tiene un estilo basado en modificar constantemente el foco atencional del niño, y nombra objetos a los que el niño no está atendiendo, afecta negativamenteal desarrollo del lenguaje.
Tomasello y sus colaboradores (Tomasello y Todd, 1983; Tomasello y Farrar, 1986), examinaron la relación entre el vocabulario del niño y la habilidad de la madre para regular los estados atencionales de su hijo. Encontraron que los padres que actuaban cambiando la interacción iniciada por el niño, tenían hijos con vocabularios más pequeños que aquellos que se adaptaban a los intereses de su hijo. Cuando las madres organizaban la interacción dirigiendo la atención del niño, en vez de seguirla, sus hijos aprendían menos nombres de objetos y más palabras personales-sociales. La sensibilidad de los padres se manifiesta en conductas como su habilidad para mantener la atención y la motivación de su hijo hacia la tarea, la cantidad de tiempo dedicada a compartir la atención, así como la capacidad para simplificar la tarea, demostrar y señalar las características relevantes de la misma, y acomodarse a los estados emocionales de los niños. Dichas variables han resultado buenos predictores de la posterior adquisición del vocabulario por el niño (Dunham y Dunham 1995; Markus, Mundy, Morales, Delgado, y Yale, 2000; Mundy y Gomes, 1998; Tomasello y Farrar, 1986; Ulvund y Smith, 1996).

Como hemos visto a lo largo de esta revisión, nuestro sistema nervioso está diseñado -y es algo que compartimos con otros primates- para orientar nuestra atención hacia la línea de la mirada de nuestro interlocutor. Sin embargo, aunque algunos estudiosos consideraban esta conducta suficiente para establecer una atención conjunta, otros consideran necesario que exista además una actividad intencional por parte de ambos. A este respecto, el modelo sociocognitivo de la atención conjunta sugiere que una vez que los niños han comprendido que sus propias intenciones les llevan a realizar determinadas conductas, están en condiciones de darse cuenta también de que las conductas de los demás obedecen asimismo a intenciones (Tomasello, Carpenter, Call, Behne y Moll, 2005). En la misma línea un estudio de Brooks y Meltzoff (2005) muestra claramente cómo el desarrollo socio-cognitivo favorece la existencia de una auténtica atención conjunta. En una situación clásica de atención conjunta como la de Butterworth (1991), los investigadores giraban la cabeza para mirar a un lado o a otro, pero en una condición lo hacían con los ojos abiertos, y en la otra con los ojos cerrados. En el estudio participaron niños de 9, 10, y 11 meses. Los resultados mostraron que mientras que en la condición "ojos abiertos" todos los niños seguían la mirada, cuando los investigadores giraban la cabeza con los ojos cerrados, sólo los más pequeños lo hacían. Los autores del estudio interpretan que esto es debido a que a partir de los 10 meses los niños saben que si alguien "mira" a un sitio con los ojos cerrados, no debe merecer la pena seguir la dirección de su mirada. Así pues, una conducta inicialmente automática se inhibe en virtud de un conocimiento de tipo social, lo que implica que los niños tienen en cuenta la intención de su interlocutor. 


\section{Conclusiones y perspectivas futuras}

Este artículo presenta una revisión de la investigación sobre la aparición y el desarrollo de la atención conjunta en la infancia, destacando su relación con la intencionalidad, la cognición social, y el lenguaje.

El presente trabajo comparte la perspectiva de que la atención conjunta es algo más que una mera coincidencia de miradas entre el niño y su madre hacia un objeto. Consideramos que este hito del desarrollo, que conlleva una serie de comportamientos, sintetizados básicamente en responder o iniciar propuestas de interacción, tiene como motor la capacidad del niño para comunicar y compartir sus intenciones sobre el mundo, pero que eso requiere el conocimiento de que su interlocutor también tiene intenciones.

Pese a que hemos presentado la secuencia de desarrollo de la atención conjunta en los niños, pensamos que la investigación debería centrarse sobre los aspectos de la interacción que resultan más relevantes para favorecer dicho desarrollo, tales como el estilo de interacción del cuidador, y la contribución de las diferencias individuales del temperamento del niño a la conformación de las diferentes formas de interacción y comunicación niño-adulto, o las diferencias individuales en la capacidad para llevar a cabo con éxito comportamientos de atención conjunta, tanto por parte del cuidador como del niño. Posteriores trabajos deberían orientarse hacia el estudio de nuevas formas de definir los comportamientos más sofisticados de atención conjunta, de manera que constituyan un paso más a los ampliamente utiliza- dos de la escala ESCS de Mundy et al. (1996). Estos aspectos nos permitirán conocer mejor la importancia de la habilidad de la atención conjunta en los niños, y sus relaciones con otros hitos de la infancia y niñez, proporcionando las herramientas necesarias a las instituciones, profesionales y padres para estimular su desarrollo.

Por ejemplo, el modelo socio-cognitivo establece algunas predicciones interesantes. Por una parte, sugiere que la atención conjunta podría ser un precedente de la Teoría de la Mente. El experimento de Brooks y Meltzoff demuestra que los niños llegan a ser capaces de inhibir una respuesta innata de orientación guiándose por la intención del interlocutor. Merece la pena profundizar en esta línea de trabajo para comprobar si el diseño de este tipo de tareas y las demandas que se le solicitan al niño son antecedentes de la presencia posterior de la teoría de la mente. Mutatis mutandis, se convierten en un tipo de interacción que puede fomentar en los niños, sobre todo en quienes tienen alguna dificultad como el autismo, un entrenamiento para tener en cuenta la perspectiva de los otros. Como han señalado Mundy et al. (2000), los niños autistas tienen más problemas para iniciar la atención conjunta que para responder a ella. Iniciar la atención conjunta requiere la interconexión entre los sistemas atencionales anterior y posterior que, significativamente, parecen ser también una de las causas de las deficiencias cognitivas del autismo (Cherkassky, Kana, Keller, y Just, 2006). Si este hecho se confirma, las deficiencias en las conductas de iniciar la atención conjunta podrían ser un indicador precoz de un posible trastorno del espectro autista.

\section{Referencias}

Adamson, L. B. (1996). Communication development during infancy.Boulder \& Co: Westview.

Adamson, L. B. y Bakeman, R. (1985). Affect and attention: Infant observed with mother and peers. Child Development, 56, 582-593.

Adamson, L. B. y Chance, S. (1998). Coordinating attention to people, objects, and symbols. En A. M. Wetherby, S. F. Warren, y J. Reichle (Eds.) Transitions in prelinguistic communication: Preintentional to intentional and presymbolic to symbolic. (pp. 15-37). Baltimore, MD: Brookes.

Adamson, L. y Bakeman, R. (1991). The development of shared attention during infancy. En R. Vasta (Ed.), Annals of Child Development, 8 (pp. 141). London: Kingsley.

Adamson, L. y McArthur, D. (1995). Joint attention, affect and culture. En C. Moore y P. Dunham (Eds.), Joint attention: Its origins and role in development (pp. 205-222). Hillsdale, New Jersey: Lawrence Erlbaum.

Adamson, L. y Russell, C. (1999).Emotion regulation and the emergence of joint attention. En Rochat, P. (Ed.), Early social cognition: Understanding others in the first month of life (pp. 281-297). Mahwah, NJ, US: Lawrence Erlbaum Associates, Inc., Publishers, 341, pp.281-297.

Awh, E., Armstrong, K., Moore, T. (2006) Visual and oculomotor selection: links, causes and implications for spatial attention. Trends in Cognitive Sciences 10, 124-130

Bakeman, R. y Adamson, L. B. (1986). Infants' conventionalized acts: Gestures and words with mothers and peers. Infant Behavior and Development, 9, 215-230.

Bakeman, R. y Adamson, L.B. (1984). Co-ordinating attention to people and objects in mother-infant and peer-infant interaction. Child Development, $55,1278-1289$.

Bang, E. y Tomasello, M. (2010). Origins of human communication. Journal of Child Language , 37(2), 470-475

Baron-Cohen, S. (1995). The eye detection detector (EDD) and the shared attention mechanism (SAM): Two cases for evolutionary psychology. En C. Moore y P. Dunham (Eds.), Joint attention: Its Origins and Role in Development (pp. 41-60). Hillsdale, New Jersey: Lawrence Erlbaum.

Bates, E. (1979). The emergence of symbols: Cognition and communication in infancy. New York, N.Y.: Academic Press.

Bates, E., Benigni, L., Bretherton, I., Camaioni, L. y Volterra, V. (1979). The emergence of symbols: Cognition and communication in infancy. New York. Academic Press.

Bates, E., Bretherton, I. y Snyder, L. (1988). From first words to grammar: Individual differences and dissociable mechanisms. New York: Cambridge University Press.

Bates, E., Camaioni, L. y Volterra, V. (1975). The acquisition of performatives prior to speech. Merril Palmer Quarterly, 21(3), 205-226.

Blacher, J. y Lauderdale, S.(2010). Do you see what I see? Joint Attention and Its Importance in Autism. The Exceptional Parent (Online,) 40(11), 3840.

Bloom, L. (1993). The transition from infancy to language: Acquiring the power of expression. Cambridge, UK: C.U.P.

Bretherton, I. (1991). Intentional communication and the development of an understanding of mind. En D. Frye y C. Moore (Eds.), Children's theories of mind: Mental states and social understanding (pp. 49-75) Hillsdale, NJ: Erlbaum.

Brooks R, Meltzoff A. (2005). The development of gaze following and its relations to language. Developmental Science. 8:535-543

Bruner, J. (1975). The ontogenesis of speech acts. Journal of Child Language, 2, $1-11$.

Bruner, J. (1977). Early social interaction and language acquisition. En H. R. Schaffer (Ed.), Studies in mother-infant interaction (pp. 271-289). New York: Academic Press.

Bruner, J. (1983). Child's talk: Learning to use language. Oxford. England: Oxford University Press.

Butterworth, G. E. (1991). The ontogeny and phylogeny of joint visual attention. En A. Whiten (Ed.), Natural theories of mind: Evolution, development and simulation of everyday mindreading (pp. 223-232). Oxford, England: Basil Blackwell. 
Butterworth, G. E. e Itakura, S. (2000). How the eyes, head and hand serve definite reference. British Journal of Developmental Psychology, 18(1), 25-50.

Butterworth, G. E. y Hopkins, B. (1989) Hand-mouth co-ordination in the newborn human infant. British Journal of Developmental Psychology, 6(4), 303-314.

Butterworth, G. y Cochran, E. (1980). Toward a mechanism of joint visual attention in human infancy. International Journal of Behavioural Development, 3, 253-272.

Butterworth, G. y Jarrett, N. (1991). What minds have in common is space: Spatial mechanisms serving joint visual attention in infancy. British Journal of Developmental Psychology, 9, 55-72.

Camaioni, L. (1993). The development of intentional communication: A reanalysis. In J. Nadel y L. Camaioni (Eds.), New perspectives in early communicative development (pp. 82-96). London, UK: Routledge.

Campbell, J. (2002). Joint attention and simulation. En Jerome Dokic and Joelle Proust (Eds.), Simulation and Knowledge of Action (pp. 241-253). Amsterdam and Philadelphia: John Benjamins.

Carpenter, M., Nagell, K. yTomasello, M. (1998). Social Cognition, joint attention, and communicative competence from 9 to 15 months of age. Monographs of the Society for Research in Child Development, 63, 4 (Serial No. 255).

Chanon, V. y Hopfinger, J. (2011). ERPs reveal similar effects of social gaze orienting and voluntary attention, and distinguish each from reflexive attention. Attention, Perception, and Psychophysics, 73:2502-2513

Cleveland, A. y Striano, T. (2007). The effects of joint attention on object processing in 4- and 9- month-old infants. Infant Behavior and Development, 30, 499-504.

Corbetta, M. (1998) Frontoparietal cortical networks for directing attention and the eye to visual locations: identical, independent, or overlapping neural systems? Proceedings National Academy of Sciences, 95, 831-838

Corkum, V. y Moore, C. (1998). Development of Joint visual Attention in Infants. En Moore y P. Dunham (Eds.), Joint attention: Its origins and role in development. (pp. 61-83). Hillsdale, New Jersey: Lawrence Erlbaum.

D'Entremont, B. y Seamans, E. (2007). Do infants need social cognition to act socially? An alternative look at infant pointing. Child Development, 78 , 723-728.

D’Entremont, B., Hains, S. M. J. y Muir, D. (1997). A demonstration of gaze following in 3 to 6 month-olds. Infant Behavior and Development, 20, 511523.

Dawson, G., Munson, J., Estes, A., Osterling, J., McPartland, J., Toth, K., Carver, L. y Abbott, R. (2002). Neurocognitive function and joint attention ability in young children with autism spectrum disorder versus developmental delay. Child Development, 73 2), 345-58.

Deák, G. O., Flom, R. y Pick, A. D. (2000). Perceptual and motivational factor affecting joint visual attention in 12- and 18-month-olds. Developmental Psychology, 36, 511-523.

Dennett, D. (1987). The intentional stance. Cambridge, MA: MIT Press, $5^{\text {th }}$ edition.

Driver, J., Davis, G., Ricciardelli, P., Kidd, P., Maxwell, E., y Baron-Cohen, S. (1999). Gaze perception triggers reflexive visuospatial orienting. Visual Cognition, 6, 509-540.

Dueker, G., Portko, S. y Zelinsky, M. (2011). Meaningful touch in naturalistic contexts: haptic input as a cue to the referent of infant directed speech. Cognitie, Creier, Comportament, 15 (4), 427

Dunham, P. y Dunham, F. (1995). Optimal social structures and adaptative infant development. En C. Moore y P.J. Dunham (Eds.), Joint Attention: Its Origins and Role in Development (pp. 159-88). Hillsdale, NJ: Lawrence Erlbaum.

Elgier, A. M., y Mustaca, A. E. (2009). Perspectivas teóricas contemporáneas sobre el gesto de señalar infantil. Avances en Psicología Latinoamericana, 27, 281-296.

Emery, N. J., Lorincz, E. N., Perret, D. I., Oram, M. W. y Baker, C. I. (1997). Gaze following and joint attention in rhesus monkeys (Macacamulatta). Journal of Comparative Psychology, 111, 286-293.

Estigarribia, B. y Clark, E. (2007). Getting and maintaining attention in talk to young children. Journal of Child Language, 34, 799-814

Fenson, L., Dale, P., Reznick, J., Bates, E., Thal, D. y Pethick, S. (1994).Variability in early communication development. Monographs of the Society of Research in Child Development, 59 (5, Serial No. 242).
Flom, R., Deák, G. O., Phill, C. G. y Pick, A. D. (2004). Nine-month-olds' shared visual attention as a function of gesture and object location. Infant Behavior and Development, 27, 181-194.

Friesen, C. K., y Kingstone, A. (2003). Abrupt onsets and gaze direction cues trigger independent reflexive attentional effects. Cognition, 87, B1B10

Goldin-Meadow, S. (2007). Pointing sets the stage for learning languageand creating language. Child Development, 78(3), 741-745.

Goodwyn, S. W., Acredolo, L. P. y Brown, C. (2000). Impact of symbolic gesturing on early language development. Journal of Nonverbal Behavior, $24,81-103$

Grassmann, S., y Tomasello, M. (2010). Young children follow pointing over words in interpreting acts of reference. Developmental Science, 13, 252-263.

Griffith, E. M., Penningotn, B. F., Wehner, E. A. y Rogers, S. J. (1999) Executive functions in young children with autism. Child Development, 70, 817-832.

Grosse, G. y Tomasello, M. (2012).Two-year-old children differentiate test questions from genuine questions. Journal of Child Language , 39(1), 192204.

Grossmann, T. y Johnson, M. H. (2010). Selective prefrontal cortex responses to joint attention in early infancy. Biology Letters. doi: 10.1098/rsbl.2009.1069

Gwenden, L. Dueker, S., Portko, M. Y Zelinsky. (2011). Meaningful Touch in Naturalistic Contexts: Haptic Input As a Cue to The Referent of Infant Directed Speech. Cognition, Brain, Behavior. An Interdisciplinary Journal. Volume XV(4), 427-448

Hanako, Y. y Burling, J. 2011). A New Perspective on Embodied Social Attention. Cognition, Brain, Behavior. An Interdisciplinary Journal. Volume XV(4), 535-552

Hobson, P. (1989). On sharing experiences. Development and Psychopathology, 1 , 197-203.

HoVman, J., y Subramaniam, B. (1995). The role of visual attention in saccadic eye movements. Percept Psychophys 57, 787-795

Jellema, T., Baker, C., Wicker, B., y Perrett, D. (2000). Neural representation for the perception of intentionality of actions. Brain and Cognition. 44, 280-302.

Kalnins, I. V. y Bruner, J. S. (1974). Infant sucking used to change the clarity of a visual display. En J. Stone, H. T. Smith, y L. B. Murphy (Eds.), The competent infant. London: Tavistock.

Kaplan, F. y Hafner, V. V. (2004). Proceedings of the $4^{\text {th }}$ International Workshop on Epigenetic Robotics. In Berthouze, L., Kozima, H., Prince, C. G., Sandini, G., Stojanov, G., Metta, G., and Balkenius, C. (Eds.), The Challenges of Joint Attention (pp. 67-74). Lund University Cognitive Science Studies 117, Genova, Italy.

Kristen, S., Sodian, B., Thoermer, C., Perst, H. (2011) Infants' Joint Attention Skills Predict Toddlers' Emerging Mental State Language. Developmental Psychology 47(5), 120-7

Laakso, M. L., Poikkeus, A. M., Katajamaki, J. y Lyytinen, P. (1999). Early intentional communication as a predictor of language development in young toddlers. First Language, 19(56), 207-231.

Lamb, M. E., Morrison, D. C. y Malkin, C. M. (1987). The development of infant social expectations in face-to-face interaction: A longitudinal study. Merrill-Palmer Quartely, 33, 241-254.

Lempers, J. D. (1979). Young children's production and comprehension of nonverbal deictic behaviours. Journal of Genetic Psychology, 135, 93-102.

Leong, D. y Bodrova, E. (2012). Assessing and Scaffolding: Make-Believe Play. Young Children 67(1), 28-34.

Leung, E. H. L. y Reingold, H. L. (1981). Development of pointing as a social gesture. Developmental Psychology, 17 (2), 215-220.

Lew, A. y Butterworth, G. E. (1997). The development of hand-mouth coordination in 2 to 5 month old infants: Similarities with reaching and grasping. Infant Behaviour and Development 20, 159-169.

Liebal, K., Carpenter, M., \& Tomasello, M. (2010). Infants' use of shared experience in declarative pointing. Infancy, 15, 545-556

Liszkowski, U. (2011). Three lines in the emergence of prelinguistic communication and social cognition. Journal of cognitive education and psychology, 10(1), 32-43.

Markus, J., Mundy, P., Morales, M., Delgado C. E. y Yaale M. (2000). Individual differences in infant skills as predictors of child-caregiver joint attention and language. Social Development, 9, 302- 315. 
Mccune, L. (2008). How children learn to learn language. Oxford: Oxford University Press.

McEvoy, R. E., Rogers, S. J. y Pennington, B. F. (1993).Executive function and social communication deficits in young autistic children. Journal of child psychology and psychiatry, 34 (4), 563-578.

Meins, E., Fernyhough, C., Arnott, B., Vittorini, L., Turner, M., Leekam, S. R. y Parkinson, K. (2011). Individual Differences in Infants' Joint Attention Behaviors with Mother and a New Social Partner. Infancy, 16.

Messer, D. J., y Vietze, P. M. (1984). Timing and transitions in motherinfant gaze. Infant Behavior and Development, 7, 167-181.

Moore, C. y Corkum, V. (1994). Social understanding at the end of the first year of life. Development Review, 14, 349-372.

Moore, C., Angelopoulos, M., y Bennet, P. (1997). The role of movement in the development of joint visual attention. Infant Behavior and Development, 20, 83-92.

Morales, M., Mundy, P. M. Delgado, C., Yale, M., Neal, A. R. y Schwartz, H. K. (2000).Gaze following, temperament, and language development in 6-month-olds: A replication and extension. Infant Behavior and Development, 23, 231-236.

Morales, M., Mundy, P. y Rojas, J. (1998). Following the direction of gaze and language development in 6-month-olds. Infant Behavior and Development, 21, 373-377.

Morales, M., Mundy, P., Delgado, C., Yale, M., Messinger, D., Neal, R., y Schwartz H. (2000). Responding to joint attention across the 6- through 24-month age period and early language acquisition. Journal of Applied Developmental Psychology. 21, 283-298

Morissette, P., Ricard, M. y Gouin-Decaire, T. (1995). Joint visual attention and pointing in infancy: A longitudinal study of comprehension. British Journal of Developmental Psychology, 13, (2), 163-177.

Mundy, P. (2003). The neural basis of social impairments in autism: The role of the dorsal medial-frontal cortex and anterior cingulate system. Journal of Child Psychology \& Psychiatry. 44, 793-809

Mundy, P. y Acra, F. (2006). Joint attention, social engagement and the development of social competence. En P. Marshall and N. Fox (Eds.), The development of social engagement neurobiological perspectives (pp. 81-117). New York: Oxford University Press.

Mundy, P. y Gomes, A. (1998). Individual differences in joint attention skill development in the second year. Infant Behavior and Development, 21, 469 82.

Mundy, P. y Sigman, M. (2006). Joint attention, social competence and developmental psychopathology. En D. Cicchetti y D. Cohen (Eds.), Developmental Psychopathology, Second Edition, Volume One: Theory and Methods, Hoboken, N.J.: Wiley.

Mundy, P., Block, J., Delgado, C., Pomares, Y., Vaughan, V. y Parlade, M. (2007) Individual differences and the development of joint attention in infancy. Child Development, 78 (3) 938-54.

Mundy, P., Hogan, A. y Doehring, P. (1996). A preliminary manual for the abridged. Early Social Communication Scales (ESCS). Recibido el 10 de abril de 2002, University of Miami-Coral Gables, Psychology Department.

Mundy, P., Kasari, C. Sigman, M. y Ruskin, E. (1995). Nonverbal communication and early language acquisition in children with Down syndrome and in normally developing children. Journal of speech and bearing research, 38, 157-167.

Muñeton, M. y Rodrigo, M. J. (2011). Functions of the Pointing Gesture in Mothers and their 12 to 36-Month-Old Children during Everyday Activities. The Spanish Journal of Psychology, Vol. 14(2), 619-629

Murphy, C. M. y Messer, D. J. (1977). Mothers, infants, and pointing: A study of gesture. En H. R. Schaffer (Ed.), Studies in mother-infant interaction (pp. 325-354). London: Academic Press.

Nichols, K., Fox, N. y Mundy, P. (2005). Joint attention, self-recognition and neurocognitive functioning. Infancy, 7, 35-52.

Olson, J. y Masur, E. (2011). Infants' gestures influence mothers' provision of object, action and internal state labels. Journal of Child Language, 38(5), 1028-54.

Olson, S. L., Bates, J. E. y Bayles K. (1984). Mother-infant interaction and the development of individual differences in children's cognitive competence. Developmental Psychology, 20, 166-179.
Ricciardelli, P., Betta, E., Pruner, S. y Turatto, M. (2009). Is there a direct link between gaze perception and joint attention behaviours? Effects of gaze contrast polarity on oculomotor behavior. Experimental Brain Research, 194, 347-357

Scaife, M. y Bruner, J. S. (1975). The capacity for joint visual attention in the infant. Nature, 253, 265-266.

Schietecatte, I., Roeyers, H. y Warreyn, P. (2011). Can infants' orientation to social stimuli predict later joint attention skills? British Journal of Developmental Psychology, 29.

Selbert, J., Hogan, A. y Mundy, P. (1982). Assessing social interactional competences: the Early Social-Communicational Scales. Infant Mental Health Journal, 3, 244-259.

Stern, D. N. (1985). The interpersonal world of the infant: A view from psychoanalysis and developmental psychology. New York: Basic Books.

Sun, J. y Rao, N. (2012). Scaffolding Interactions with Preschool Children: Comparisons between Chinese Mothers and Teachers across Different Tasks. Merrill - Palmer Quarterly , 58, 110-140.

Tomasello M, Carpenter M, Call J, Behne T, Moll H. (2005). Understanding and sharing intentions: The origins of cultural cognition. Bebavioral and Brain Sciences, 28, 675-735

Tomasello, M. (1988). The role of joint attention in early language development. Language Sciences, 11, 69-88.

Tomasello, M. (1995). Joint attention as social cognition. En C. Moore y P. Dunham (Eds.), Joint attention: Its origins and role in development (pp. 103130). Hillsdale, NJ: Lawrence Erlbaum.

Tomasello, M. (1998). Cognitive linguistics. En W. Bechtel y G. Graham (Eds.), A Companion to Cognitive Science. Basil Blackwell.

Tomasello, M. y Carpenter, M. (2007). Shared intentionality. Developmental Science 10(1), 121-125.

Tomasello, M. y Farrar, J. (1986). Joint attention and early language. Child Development, 57, 1454-63.

Tomasello, M. y Tood, J. (1983). Joint attention and lexical acquisition style. First Language, 4, 197-212.

Tomasello, M., Carpenter, M. y Lizskowski, U. (2007). A new look at infant pointing. Child Development, 78, 705-722.

Tomasello, M., Carpenter, M., Call, J., Behne, T. y Moll, H. (2005). Understanding and sharing intentions: The origins of cultural cognition. Behav ioral and Brain Sciences, 28(5), 675-91.

Tremblay, H. y Rovira, K. (2007). Joint visual attention and Triangular Engagement at 3 and 6 months. Infant Behavior and Development. 30(2), 366379

Trevarthen, C. (1990). Growth and education in the hemispheres. En C. Trevarthen (Ed.), Brain circuits and functions of the mind: Essays in honour of Roger W. Sperry (pp. 334-363). Cambridge: Cambridge University Press.

Tronick, E. Z. (1989). Emotions and emotional communication in infants. American Psychologist, 44, 112-119.

Ulvund, S. E. y Smith, L. (1996). The predictive validity of nonverbal communicative skills in infants with perinatal hazards. Infant Behavior and Development, 19, 441-449.

Vaughan, A., Mundy, P., Acra, C., Block, J., Delgado, C., Parlade, M., Neal A., Meyer, J. y Pomares, Y. (2007). Infant joint attention, temperament, and social competence in preschool children. Child Development, 78, 1, 53-69.

Vigotsky, L. S. (1964). Pensamiento y Lenguaje. Buenos Aires, Editorial Lautaro.

Von Hofsten, C. (1990). Development of manipulation action in infancy. En H. Bloch y B.I. Berthental (Eds.), Sensory-motor organizations and development in infancy and early childhood (pp. 33-43). Dordrecht: Kluwer.

Werner, H. y Kaplan, B. (1963). Symbol formation: An organismic-developmental approach to language and the expression of thought. New York: Wiley.

Williams, J., Waiter, G., Perra, O., Perrett, D., y Whiten, A. (2005). An fMR study of joint attention experience. NeuroImage. 25, 133-140.

Woodward, A. L. (2003). Infant's developing understanding of the link between looker and object. Developmental Science, 6, 297-311.

Yoshida, H. y Burling, J. (2011). A new perspective on Embodied social attention. Cognition, Brain, Behavior, 15, (4), 535-552

(Articulo recibido: 04-11-2011, revisado: 27-03-2012, aceptado: 24-05-2012) 